\title{
Preliminary Monte Carlo Investigation of Using Ir-192 as the Source for Real Time Imaging Purpose
}

\author{
Chengyu Shi' ${ }^{1}$ Brian Wang² \\ ${ }^{1}$ Department of Medical Physics, Memorial Sloan Kettering Cancer Center, New York, NY, USA \\ ${ }^{2}$ Department of Radiation Oncology, James Brown Cancer Center, The University of Louisville, Louisville, KY, USA \\ Email: shic@mskcc.org, brian.wang@louisville.edu
}

How to cite this paper: Shi, C.Y. and Wang, B. (2017) Preliminary Monte Carlo Investigation of Using Ir-192 as the Source for Real Time Imaging Purpose. International Journal of Medical Physics, Clinical Engineering and Radiation Oncology, 6, 21-30.

https://doi.org/10.4236/ijmpcero.2017.61003

Received: November 25, 2016

Accepted: January 8, 2017

Published: January 12, 2017

Copyright $\odot 2017$ by authors and Scientific Research Publishing Inc. This work is licensed under the Creative Commons Attribution International License (CC BY 4.0).

http://creativecommons.org/licenses/by/4.0/

\begin{abstract}
The purpose of this study is to investigate the potential use of Ir-192 as the source for real time imaging during HDR (High Dose Rate) brachytherapy treatment. Phantom measurement was performed to determine outside of the body dose. Monte Carlo code, EGSnrcMP egs_inprz, was used for the simulation to calculate the outside of the body $\mathrm{x}$-ray signal for $\mathrm{CT}$ reconstruction. Matlab code was developed to reconstruct the Ir-192 source and for 3D visualization in order to assess reconstructed CT resolution, signal-to-noise ratio, and imaging dose information. The measured dose was $0.67 \pm 0.04 \mathrm{cGy}$, which was comparable to the Monte Carlo simulation result $0.71 \pm 0.20 \mathrm{cGy}$. The reconstructed source diameter dimension was $1.3 \mathrm{~mm}$ compared with 1.1 $\mathrm{mm}$ for the real source dimension. The signal-to-noise ratio was $19.91 \mathrm{db}$ following de-noising. Source position was within a $1 \mathrm{~mm}$ difference between programmed and simulated results. Although the Ir-192 signal is weak for CT imaging, it is possible to use it as a CT imaging $\mathrm{x}$-ray source for HDR treatment localization, verification and dosimetry purposes. Further study is needed for the detailed design of an outside of the body CT-like device for use in brachytherapy imaging.
\end{abstract}

\section{Keywords}

Monte Carlo, Ir-192, HDR, Imaging

\section{Introduction}

With the goal of sparing organs at risk (OARs), new radiation therapy techniques have been developed in the past two decades to treat the target while avoiding healthy tissues. Intensity Modulated Radiation Therapy (IMRT) tech- 
nique has been widely applied to external beam radiation therapy. In order to ensure precision and accuracy of treatment, IMRT requires combined use with Imaging Guided Radiation Therapy (IGRT). However, in brachytherapy, the implementation of IMRT is delayed due to treatment space and physical limitations. Several studies have tried to apply IMRT concept to brachytherapy. In 2002, Ebert investigated the use of Intensity-Modulated Brachytherapy (IMBT) with radially asymmetric internally applied radiation sources [1]. In 2008, Hiatt et al. introduced the concept of Intensity Modulated Electronic Brachytherapy (IMEB) [2], and then in 2009 further explored depth dose modulation electronic brachytherapy to spare skin in intracavitary breast treatment [3]. In 2010, Shi et al. used dosimetry algorithm and inverse treatment planning to calculate threedimensional (3D) IMBT dose distributions [4]. All these studies focused on the IMBT concept and dosimetry. Recently, Liu et al. developed a rotating-shield device in 2013 and later modified it for application in different brachytherapy treatments [5]-[10], further expanding IMBT's clinical capabilities. Similar to IMRT's dependence on IGRT for external beam treatment, IMBT also relies on imaging prior to treatment, during treatment, and even following treatment with verification. Brachytherapy treatment also requires imaging guidance or realtime imaging for localization and verification purposes. Image guidance is actually more important in brachytherapy than external treatment due to the large dose variation as a result of applicator position uncertainty. Several studies showed there is approximately a $5 \%$ change in dosimetry for the target and nearby critical structures per $\mathrm{mm}$ of applicator shift [11] [12]. In a recent review of HDR (High Dose Rate) safety, authors reported about 8 events per 33,000 treatments each year [13]. The causes of these events were mostly due to wrong length, distance, or applicator. Therefore, imaging verification during treatment is important for preventing adverse events.

Real-time monitoring of applicator position has been explored with use of infrared markers [14], but this technique is burdened by bulky equipment and potential marker shifts relative to the applicators. Other existing imaging modalities, such as ultrasound, MRI (Magnetic Resonance Imaging), and C-arm x-ray, CT (Computed Tomography) either need an extra imaging source, which is not convenient in brachytherapy treatment, or lack real-time imaging potential. There are many potential benefits if the Ir-192 source itself for HDR treatment can be used as the imaging source and if a special CT type detector for imaging can be designed. This could reduce unnecessary $\mathrm{x}$-ray sources and exposure, be performed in real-time, and provide inherent source localization information. Safavi-Naeini el al. developed a BrachyView in-body imaging system for HDR prostate brachytherapy in 2013 and 2015 [15] [16] [17], which was capable of providing imaging, localization and dose validation. However, due to physical space limitations, the device may not be suitable for all types of HDR treatment.

In this study, we investigated the potential of using Ir-192 as the CT source for brachytherapy imaging purposes. Monte Carlo simulation was carried out to study the accuracy of localization with the Ir-192 source and to assess imaging 
resolution, signal-to-noise ratio, imaging dose information.

\section{Materials and Methods}

\subsection{Phantom and Treatment Planning}

An ACR (American College of Radiology) CT accreditation adult abdomen phantom (diameter $=16 \mathrm{~cm}$ ) was used for this study and is shown in Figure 1(a) and Figure 1(b). The phantom was scanned with a GE (General Electric Company, Milwaukee, Wisconsin, USA) 4-slice LightSpeed CT scanner using a brachytherapy protocol $(1.25 \mathrm{~mm}$ CT slice thickness, $50 \mathrm{~cm}$ field-of-view (FOV)). Images were then transferred to an Oncentra treatment planning system (Elekta Medical System Ltd., Stockholm, Sweden. version 4.3). A treatment plan was developed with a $3.0 \mathrm{~cm}$ diameter cylinder simulated. The prescription was $500 \mathrm{cGy}$ per fraction at $5 \mathrm{~mm}$ above the cylinder surface. Thus, $2.0 \mathrm{~cm}$ away from the center in the radius direction would receive $500 \mathrm{cGy}$ per fraction. The treatment plan was then transferred to the microSelectron HDR unit. A Fluke (Fluke Electronics Corporation, Everett, WA) Model $451 \mathrm{P}$ pressurized ion chamber survey meter was placed with the detector sensitive region touching the phantom surface for dose rate measurement. The University of Wisconsin Accredited Dosimetry Calibration Laboratory calibrated the survey meter with $\pm 6.5 \%$ uncertainty. Figure 1 shows the CT scanning and treatment setup for the adult abdomen phantom. The purpose of this phantom study is to get the range of dose rate on the phantom surface and to calculate the calibration factor for the following Monte Carlo simulation to convert fluence to absolute dose per simulated particle.

\subsection{Monte Carlo (MC) Simulation}

The EGSnrcMP egs_inprz (version 1.0) program (National Research and Council Canada) was used for the Monte Carlo simulation. EGSnrcMP egs_inprz is a graphic user interface (GUI) for the NRC RZ user-codes

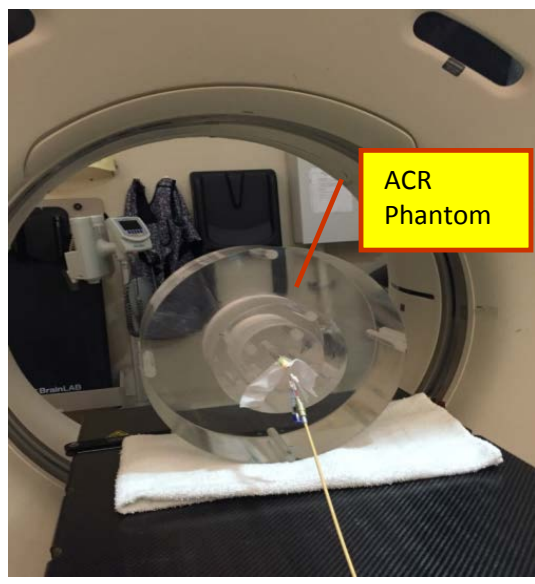

(a)

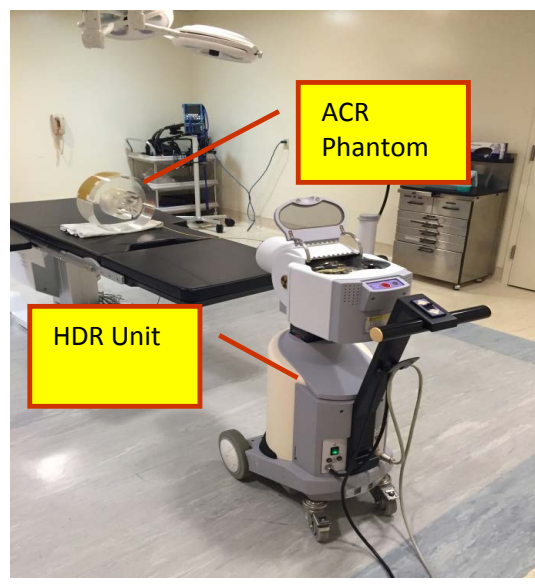

(b)

Figure 1. (a) CT scan of the adult abdomen phantom with source position simulator, (b) treatment setup for the phantom. 
(http://nrc-cnrc.github.io/EGSnrc/doc/pirs801-egsinprz.pdf). DOSRZnrc user code was selected. The adult abdomen phantom was simulated with PMMA materials and the Ir-192 microSelectron spectrum was chosen for the simulation. Uniform isotropically radiating disk of finite size was used and the settings for source simulation were: RMINBM $=0$, RBEAM $=0.06, \mathrm{ZSMIN}=5.0$ and ZSMAX = 5.35. The Ir-192 Source dimension (diameter and active length) information followed the paper published by Mowlavi et al. in 2008 [18]. In total, $10^{9}$ particles were simulated with ECUT $=0.521 \mathrm{MeV}$ and PCUT $=0.01 \mathrm{MeV}$ settings. Figure 2(a) depicts the geometry of the simulation. Longitudinal and radius profiles for the simulation were recorded. The $3 \mathrm{~mm}$ margin outside of the diameter $=16 \mathrm{~cm}$ was assumed to be detector region and used for recording the CT-like device detector signal (here is the deposited dose information) purpose.

\subsection{Post-Processing of the MC Results}

In-house Matlab (R2010a, The MathWorks, Natick, MA, USA) code was written to post-process the simulated results. The inverse radon transform ("iradon" function in Matlab) was performed and the 2D CT image was generated. The inverse radon transform will reconstruct the image from the projection data in the $2 \mathrm{D}$ array. A de-noising technique with wavelet decomposition at level 5 and soft thresholding ("wavedec2" function in Matlab with $\mathrm{N}=5$ and wname = "db5") was performed on original reconstructed 2D image to get better image quality. The wavedec 2 function will return the wavelet decomposition of the image at certain level. With the same inverse radon transform, de-noised image showed better reconstructed result.

\section{Results}

\subsection{Measurement vs. Monte Carlo Simulation for Absolute Dosimetry}

The measured dose rate using the $451 \mathrm{P}$ survey meter was $8.3 \mathrm{R} / \mathrm{h}$ (the apparent source activity was $8.11 \mathrm{Ci}$ at the measurement time), which was converted to air dose using the factor $0.877 \mathrm{cGy} / \mathrm{R}$ and a treatment time of $332.3 \mathrm{~s}$. The measured dose was $0.67 \mathrm{cGy}$. If $\pm 6.5 \%$ uncertainty was taken into account, the measured result was then $0.67 \pm 0.04 \mathrm{cGy}$. The Monte Carlo simulation result was then calibrated at $2.0 \mathrm{~cm}$ away from the phantom center receiving $500 \mathrm{cGy}$. The calibration factor was $2 \times 10^{13}$ particles/Gy. The dose was $0.71 \pm 0.20 \mathrm{cGy}$ at a radius distance of $16.3 \mathrm{~cm}$, which was $5 \%$ higher than the measured result. Factors for the difference between measurements and simulations include the survey meter's calibration uncertainty and the Monte Carlo simulation's uncertainty of $\pm 28.0 \%$ @ depth $16.3 \mathrm{~cm}$ due to the weak signal of the brachytherapy source. However, the measurement was still in the simulation range. Please note that the Monte Carlo uncertainty is independent of the source activity. The source activity was used for deriving the calibration factor for Monte Carlo simulation in order to get absolute dose information. If the source is weaker, the delivery time for the measurement will be longer, but the integral dose will be the same. 


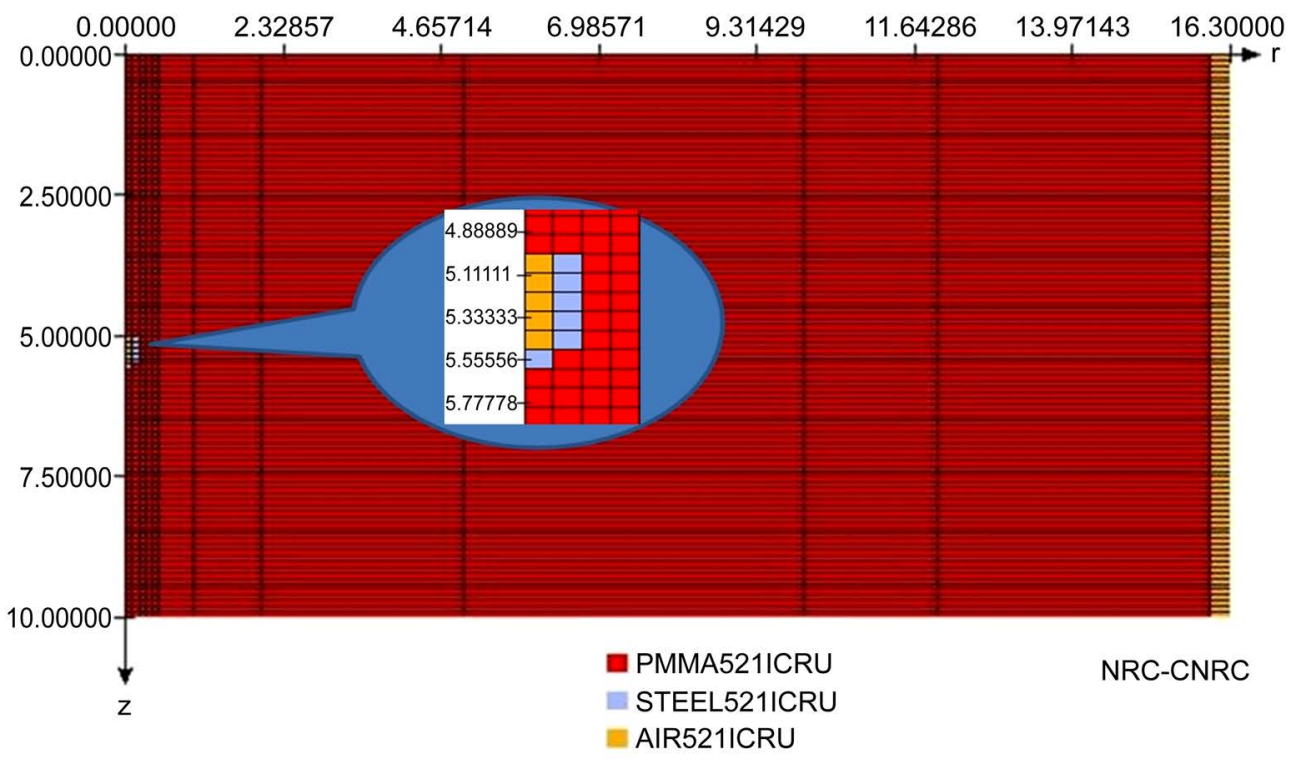

(a)

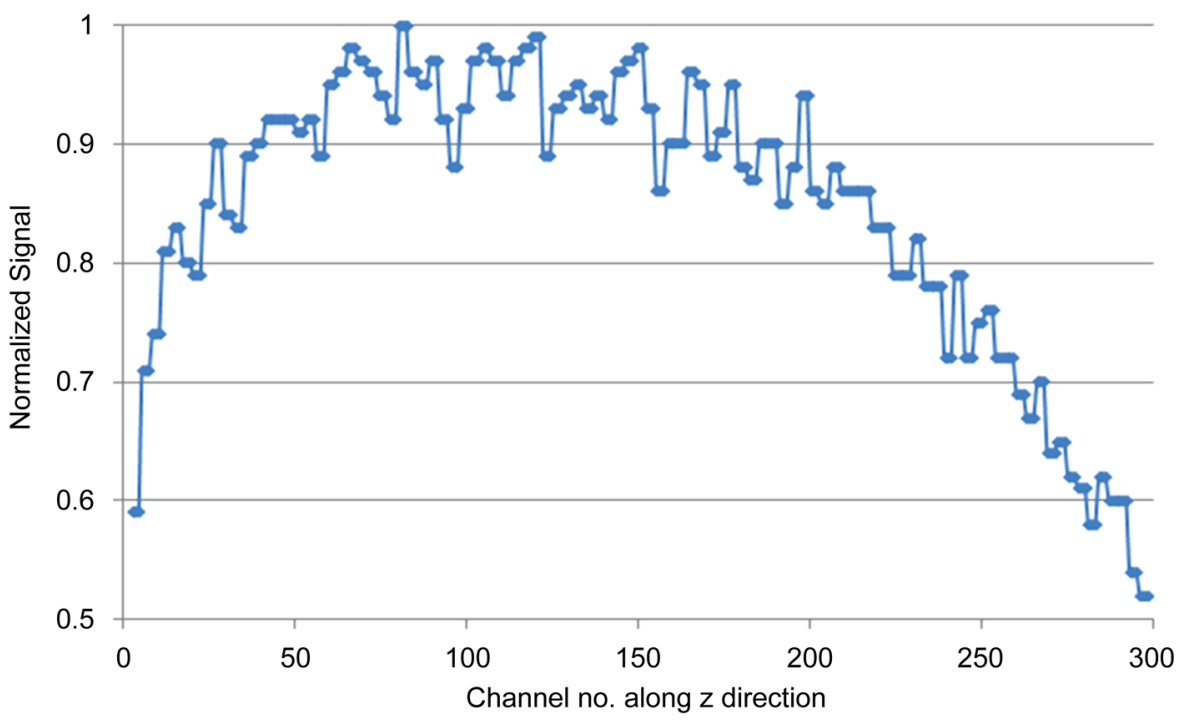

(b)

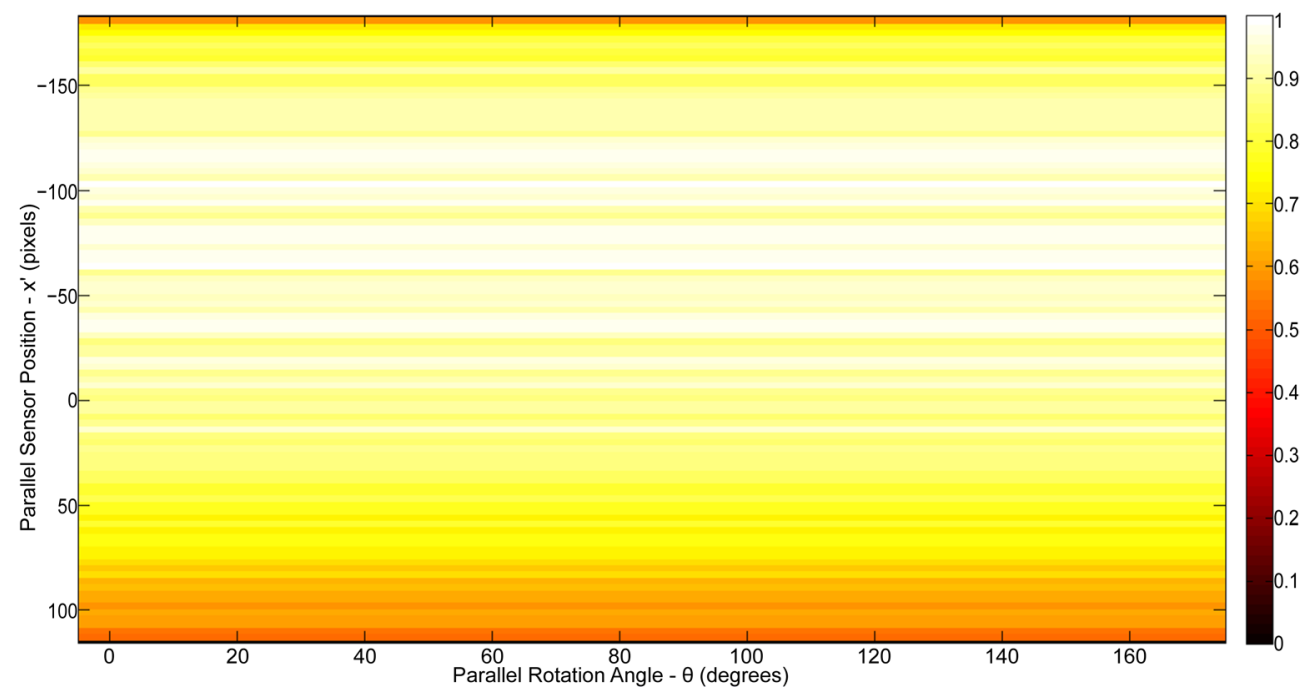

(c) 


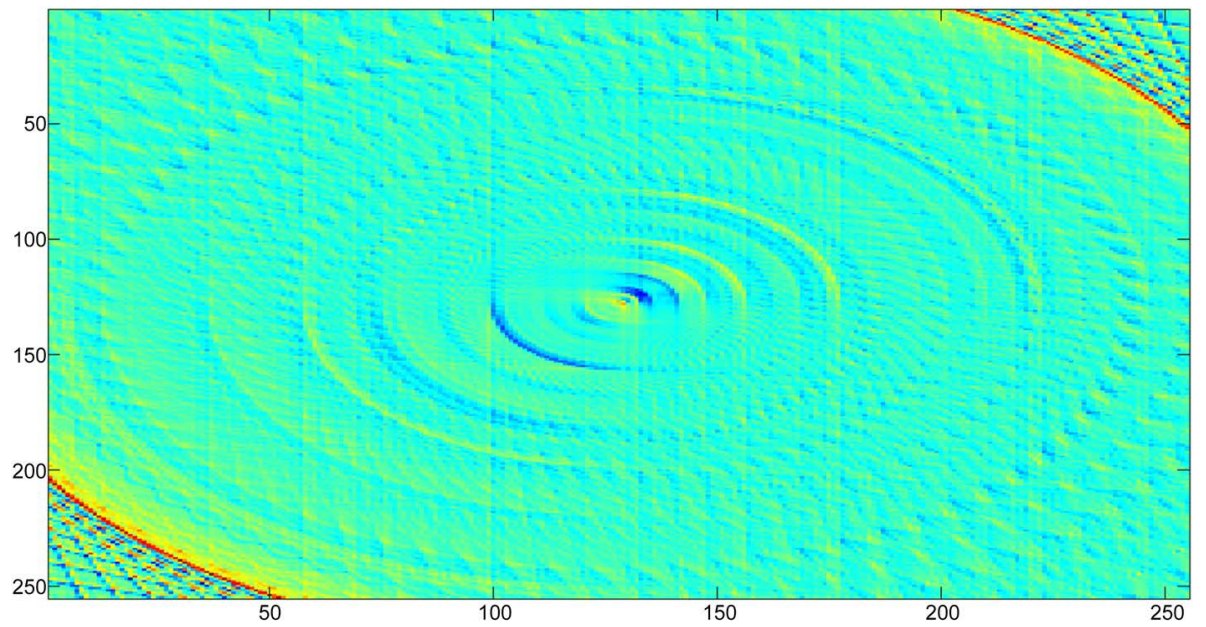

(d)

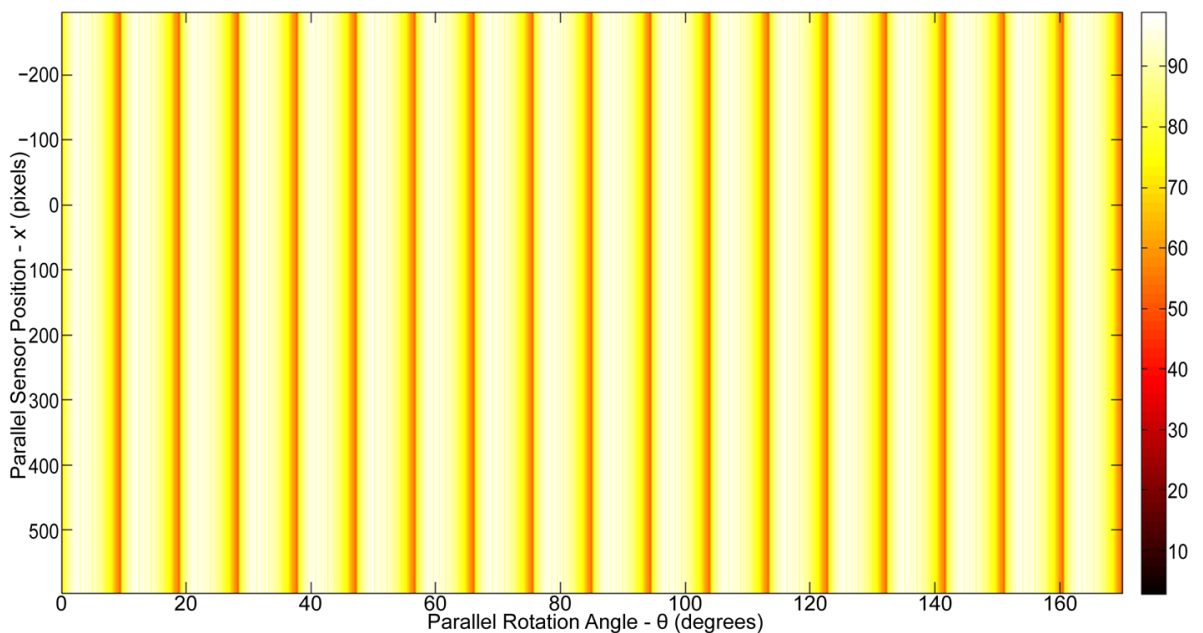

(e)

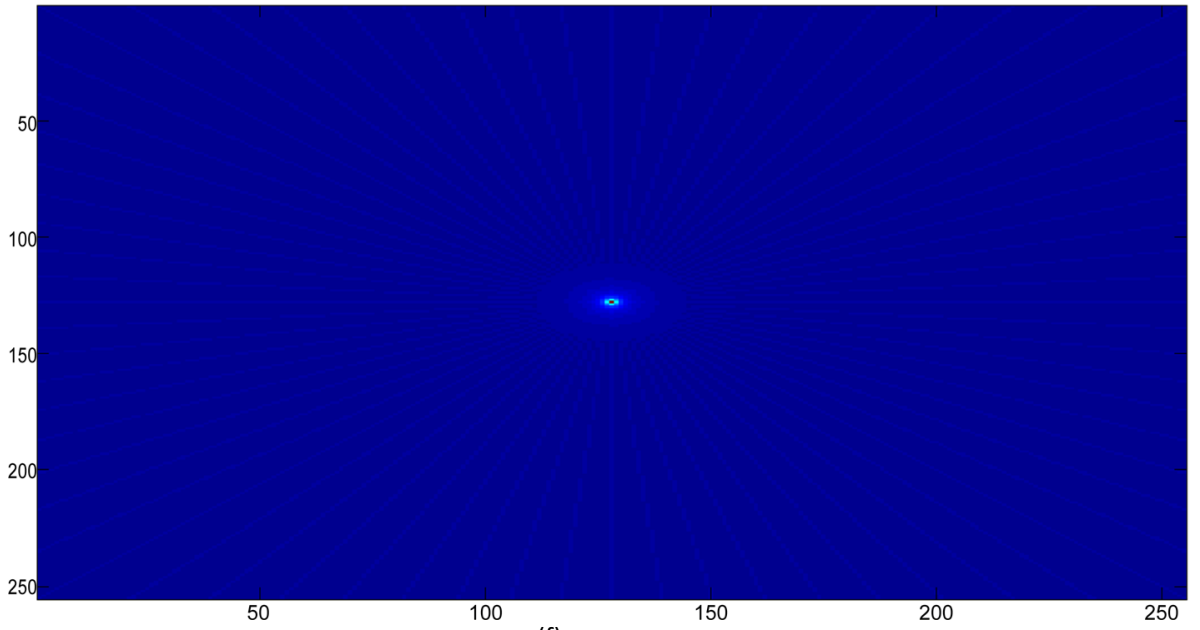

(f)

Figure 2. (a) Monte Carlo simulation geometry; (b) Detector response at the radius distance $16.3 \mathrm{~cm}$, the channel number is corresponding to the $\mathrm{z}$ direction in (a) and the signal is normalized dose deposited at the region $16.0 \mathrm{~cm}-16.3 \mathrm{~cm}$; (c) Raw projection data plot. The parallel rotation angle is corresponding to angle along the $\mathrm{z}$ direction in (a) and the parallel sensor position is corresponding to signal response in $r$ the direction. (d) Reconstructed CT image from raw projection (signal c); (e) Projection data plot with de-noising; (f) Reconstructed CT image with de-noising (signal e). 


\subsection{Source Position, Dimension Comparison and Signal-to-Noise Ratio}

Figure 2(b) shows the profile of one normalized signal result at a distance of $16.0 \mathrm{~cm}$ to $16.3 \mathrm{~cm}$ along the $\mathrm{z}$ direction. Figure 2(c) shows the original 2D CT sinogram image and Figure 2(d) shows the re-constructed image. Figure 2(e) shows the de-noised 2D CT sinogram image and Figure 2(f) shows the re-constructed image of Figure 2(e). The 2D CT was constructed using a dimension of $255 \times 255$. If $50 \%$ of the maximum signal was considered to be source diameter boundary, the source diameter dimension was then $1.3 \mathrm{~mm}(2 \times 160 \mathrm{~mm} / 255$ pixel $\times 1$ pixel), which is comparable to the $1.1 \mathrm{~mm}$ diameter reported by Mowlavi et al. in 2008 [18]. If we use the de-noised image shown in Figure 2(d) for comparison, the signal to noise ratio would be $19.91 \mathrm{db}$. Histogram plots for Figure 2(d) and Figure 2(f) show a clear improvement in signal to noise ratios and are presented in Figure 3(a) and Figure 3(b).

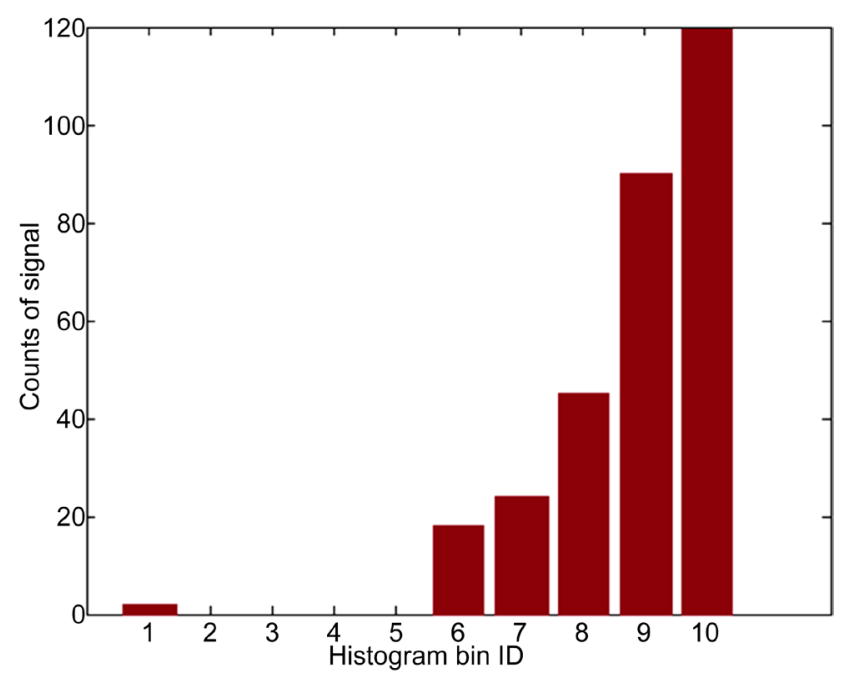

(a)

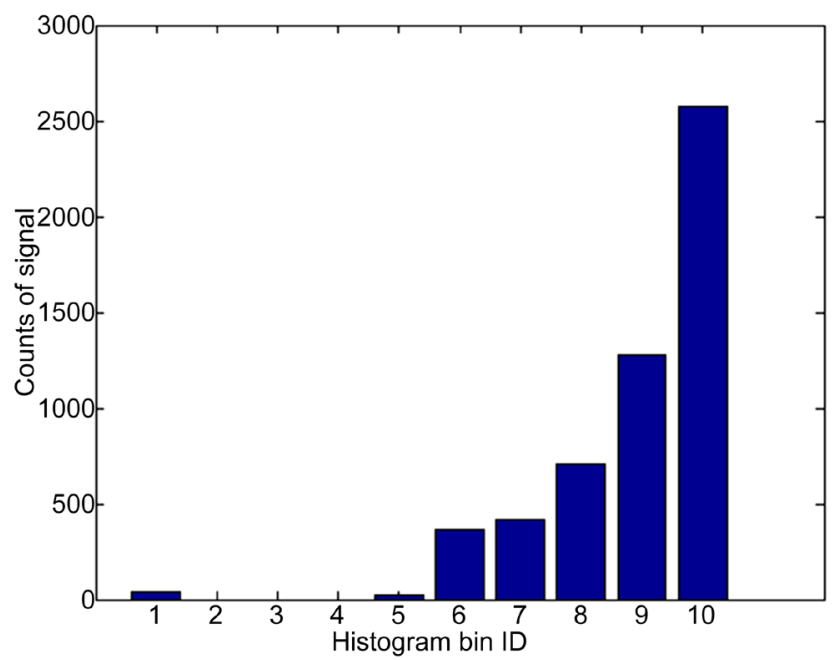

(b)

Figure 3. (a) Histogram plot for Figure 2(d), (b) histogram plot for Figure 2(f). A 20 -fold improvement was observed with the de-noising technique. 
A $2^{\text {nd }}$ Monte Carlo simulation was performed with the source retracted by 5 $\mathrm{cm}$. The results were merged with the first simulation to simulate two source dwell positions. The magnitude of the $2^{\text {nd }}$ simulation was weighted at $30 \%$ of the first simulation in order to differentiate the two signals. Figure 4 shows the dose/particle plot for the simulation of the two differentially weighted dwell positions. The second source center position calculated from the Monte Carlo simulation was $50.2 \mathrm{~mm}$ compared to the programmed center position of 50.175 $\mathrm{mm}$. The sub-millimeter accuracy of results clearly illustrates correct source position and relative weight.

\section{Discussions and Summary}

Even though the signal response is low compared to the prescribed dose $(0.672 / 500=0.13 \%)$, it is still possible to use the Ir-192 source as a radiation source for CT-like imaging. With proper signal processing, this could offer localization and verification information. Our Monte Carlo simulation results show that the image can offer correct source dose, position, and dimension information despite of relative high uncertainties.

Currently, there are few publications on the use of the Ir-192 source itself as an imaging source. Safavi-Naeini et al. designed a BrachyView in vivo imaging device for brachytherapy [15] [16] [17], which can achieve sub-millimeter accuracy with an equivalent acquisition time of $0.5 \mathrm{~s}$ and their results support the use of the Ir-192 source as an imaging source. However, since BrachyView is an inbody device, its application is limited in other treatment sites, such as with breast HDR. For the real device design, the publication by Duan (2010) showed a

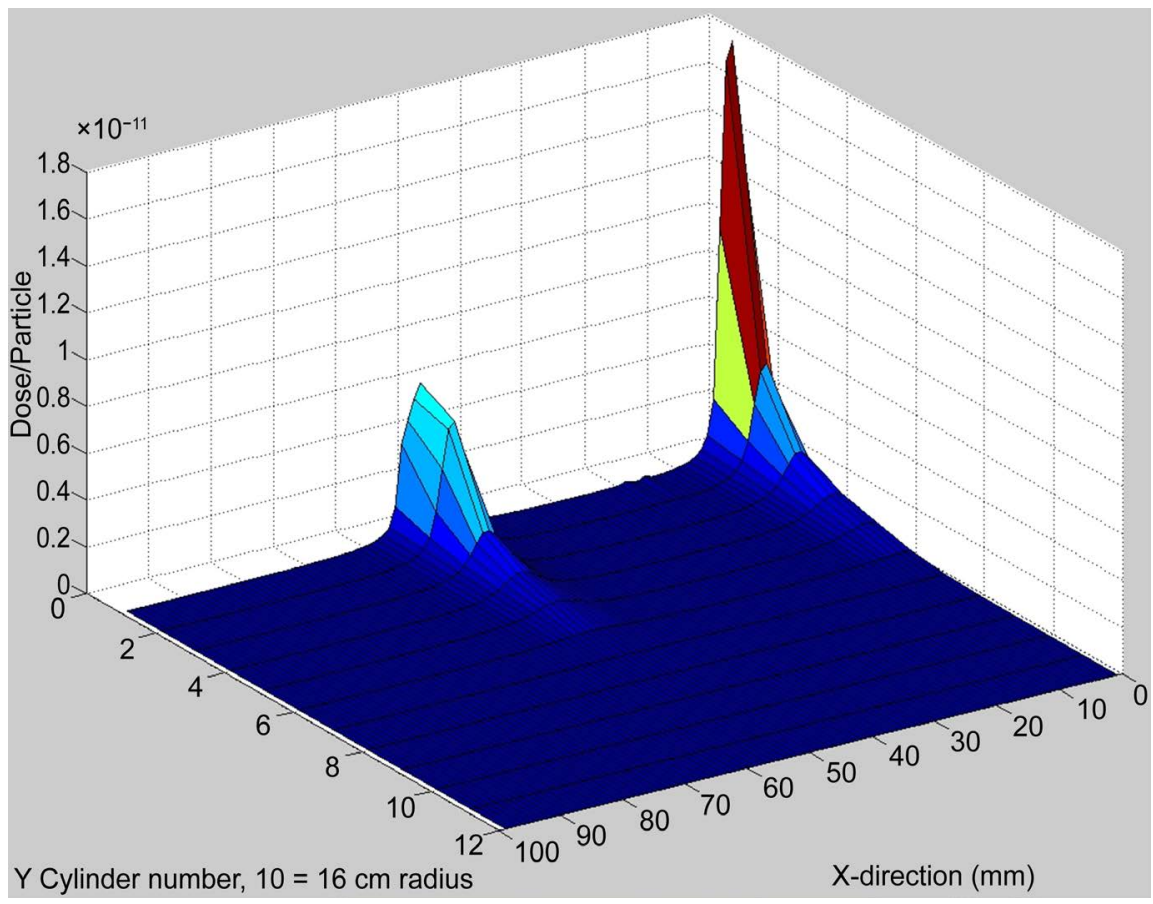

Figure 4. Simulation of two differentially weighted dwell positions. Where $\mathrm{X}$ direction is along Figure 2(a) $\mathrm{z}$ direction and $\mathrm{Y}$ direction is along Figure 2(a) r direction. 
$0.5 \mathrm{~mm}$ pinhole camera could resolve source position separation as small as 1 $\mathrm{mm}[19]$.

Our goal is to design an outside of the body CT-like device and to achieve similar imaging and dose information as was done with BrachyView. Further study is still needed for the detailed design of an out of the body CT-like device for brachytherapy imaging, but this preliminary study provides a proof of a concept for Monte Carlo simulations and also provides guidelines for future device design.

\section{Acknowledgements}

This research was funded in part through the NIH/NCI Cancer Center Support Grant P30 CA008748.

\section{References}

[1] Ebert, M.A. (2002) Possibilities for Intensity-Modulated Brachytherapy: Technical Limitations on the Use of Non-Isotropic Sources. Physics in Medicine and Biology, 47, 2495-509. https://doi.org/10.1088/0031-9155/47/14/309

[2] Hiatt, J., Hepel, J., Carol, M., Cardarelli, G., Wazer, D. and Sternick, E. (2008) Physical Principles of Intensity Modulated Electronic Brachytherapy (IMEB). Medical Physics, 35, 2732. https://doi.org/10.1118/1.2961778

[3] Hiatt, J., Segala, J., Gardarelli, G. and Sternick, E. (2009) The Utility of Depth Dose Modulation (DDM) for Electronic Brachytherapy. Medical Physics, 36, 2423. https://doi.org/10.1118/1.3181075

[4] Shi, C.Y., Guo, B.Q., Cheng, C.Y., Esquivel, C., Eng, T. and Papanikolaou, N. (2010) Three Dimensional Intensity Modulated Brachytherapy (IMBT): Dosimetry Algorithm and Inverse Treatment Planning. Medical Physics, 37, 3725-3737. https://doi.org/10.1118/1.3456598

[5] Liu, Y., Flynn, R.T., Kim, Y., Yang, W. and Wu, X. (2013) Dynamic Rotating-Shield Brachytherapy. Medical Physics, 40, Article ID: 121703. https://doi.org/10.1118/1.4828778

[6] Liu, Y., Flynn, R.T., Yang, W., Kim, Y., Bhatia, S.K., Sun, W. and Wu, X. (2013) Rapid Emission Angle Selection for Rotating-Shield Brachytherapy. Medical Physics, 40, Article ID: 051720. https://doi.org/10.1118/1.4802750

[7] Liu, Y., Flynn, R.T., Kim, Y. and Wu, X. (2014) Asymmetric Dose-Volume Optimization with Smoothness Control for Rotating-Shield Brachytherapy. Medical Physics, 41, Article ID: 111709. https://doi.org/10.1118/1.4897617

[8] Liu, Y., Flynn, R.T., Kim, Y., Dadkhah, H., Bhatia, S.K., Buatti, J.M., Xu, W. and Wu, X. (2015) Paddle-Based Rotating-Shield Brachytherapy. Medical Physics, 42, 5992-6003. https://doi.org/10.1118/1.4930807

[9] Adams, Q.E., Xu, J., Breitbach, E.K., Li, X., Enger, S.A., Rochey, W.R., Kim, Y., Wu, X. and Flynn, R.T. (2014) Interstitial Rotating Shield Brachytherapy for Prostate Cancer. Medical Physics, 41, Article ID: 051703. https://doi.org/10.1118/1.4870441

[10] Dadkhah, H., Kim, Y., Wu, X. and Flynn, R.T. (2015) Multihelix Rotating Shield Brachytherapy for Cervical Cancer. Medical Physics, 42, 6579-6588. https://doi.org/10.1118/1.4933244

[11] Tanderup, K., Hellebust, T.P., Lang, S., Granfeldt, J., Potter, R., Lindegaard, J.C. and Kirisits, C. (2008) Consequences of Random and Systematic Reconstruction Uncer- 
tainties in 3D Image Based Brachytherapy in Cervical Cancer. Radiotherapy and Oncology, 89, 156-163. https://doi.org/10.1016/j.radonc.2008.06.010

[12] Schindel, J., Zhang, W., Bhatia, S.K., Sun, W. and Kim, Y. (2013) Dosimetric Impacts of Applicator Displacements and Applicator Reconstruction-Uncertainties on 3D Image-Guided Brachytherapy for Cervical Cancer. Journal of Contemporary Brachytherapy, 5, 250-257. https://doi.org/10.5114/jcb.2013.39453

[13] Thomadsen, B.R., Erickson, B.A., Eifel, P.J., Hsu, I.C., Patel, R.R., Petereit, G.D., Fraass, B.A. and Rivard, M.J. (2014) A Review of Safety, Quality Management and Practice Guidelines for High-Dose-Rate Brachytherapy: Executive Summary. Practical Radiation Oncology 4, 65-70. https://doi.org/10.1016/j.prro.2013.12.005

[14] Xia, J., Waldron, T. and Kim, Y. (2014) A Real-Time Applicator Position Monitoring System for Gynecologic Intracavitary Brachytherapy. Medical Physics, 41, Article ID: 011703. https://doi.org/10.1118/1.4842555

[15] Petasecca, M., Loo, K.J., Safavi-Naeini, M., Han, Z., Metcalfe, P.E., Meikle, S., Pospisil, S., Jakubek, J., Bucci, J.A., Zaider, M., Lerch, M.L.F., Qi, Y. and Rosenfeld, A.B. (2013) BrachyView: Proof-of-Principle of a Novel In-Body Gamma Camera for Low Dose-Rate Prostate Brachytherapy. Medical Physics, 40, Article ID: 041709. https://doi.org/10.1118/1.4794487

[16] Safavi-Naeini, M., Han, Z., Cutajar, D., Guatelli, S., Petasecca, M., Lerch, M.L.F., Franklin, D.R., Jakubek, J., Pospisil, S., Bucci, J., Zaider, M. and Rosenfeld, A.B. (2013) BrachyView, A Novel Inbody Imaging System for HDR Prostate Brachytherapy: Design and Monte Carlo Feasibility Study. Medical Physics, 40, Article ID: 071715. https://doi.org/10.1118/1.4808360

[17] Safavi-Naeini, M., Han, Z., Alnaghy, S., Cutajar, D., Petasecca, M., Lerch, M.L.F., Franklin, D.R., Bucci, J., Carrara, M., Zaider, M. and Rosenfeld, A.B. (2015) BrachyView, A Novel Inbody Imaging System for HDR Prostate Brachytherapy: Experimental Evaluation. Medical Physics, 42, 7098. https://doi.org/10.1118/1.4935866

[18] Mowlavi, A.A., Cupardo, F. and Severgnini, M. (2008) Monte Carlo and Experimental Relative Dose Determination for an Iridium-192 Source in Water Phantom. International Journal of Radiation Research, 6, 37-42. http://ijrr.com/article-1-377-en.html

[19] Duan, J., Macey, D.J., Pareek, P.N. and Brezovich, I.A. (2001) Real-Time Monitoring and Verification of in Vivo High Dose Rate Brachytherapy Using a Pinhole Camera. Medical Physics, 28, 167-173. https://doi.org/10.1118/1.1339882 
Submit or recommend next manuscript to SCIRP and we will provide best service for you:

Accepting pre-submission inquiries through Email, Facebook, LinkedIn, Twitter, etc. A wide selection of journals (inclusive of 9 subjects, more than 200 journals)

Providing 24-hour high-quality service

User-friendly online submission system

Fair and swift peer-review system

Efficient typesetting and proofreading procedure

Display of the result of downloads and visits, as well as the number of cited articles Maximum dissemination of your research work

Submit your manuscript at: http://papersubmission.scirp.org/

Or contact ijmpcero@scirp.org 